\title{
Temperature optimization of MOX sensor arrays for odorant discrimination
}

\author{
J. Fonollosa ${ }^{1,2}$, L. Fernandez ${ }^{1,2}$, A. Gutierrez-Galvez ${ }^{1,2}, \underline{\text { S. Marco }}^{1,2}$, \\ ${ }^{1}$ Institute for Bioengineering of Catalonia (IBEC). Baldiri Reixac 4-8, 08028 Barcelona, Spain \\ ${ }^{2}$ Department of Electronics, University of Barcelona, Martí i Franqués 1, 08028-Barcelona, Spain \\ smarco@ibecbarcelona.eu
}

\begin{abstract}
:
We present a methodology based on Information theory tools to optimize the operating temperatures of metal-oxide (MOX) gas sensor arrays and maximize the ability of the system in odor discrimination tasks. We have demonstrated the feasibility of the method by optimizing the temperatures of a foursensor array for an effective discrimination of four odorants.

We measured the resistance of four MOX sensors at different operating temperatures when exposed to different concentration levels of ethanol, acetone, 2-butanone and acetic acid. Based on the acquired data we built complete models to shape the responses of the sensors according to the gas exposure conditions and the operating temperature. We applied the Mutual Information (MI) theory to quantify the ability of the sensor array to determine the identity of the stimuli regardless of its concentration, and thereby, select the optimum operation temperature for each sensor.
\end{abstract}

Key words: Artificial Olfaction, sensor array optimization, Mutual Information, MOX gas sensor, odor discrimination

\section{Introduction}

In recent years metal-oxide (MOX) gas sensors have found a wide range of applications [1] due to its high sensitivity, fast response and costeffective design. However, MOX sensors show low selectivity and suffer from a lack of reproducibility $[2,3]$.

To enhance sensor selectivity, the operating temperature is usually modulated during volatile exposition by means of an electrically insulated heater placed next to the sensing layer and controlled by its own electronic circuit. In fact, the temperature of the sensing layer changes significantly the sensor sensitivity and a sensor operated at different temperatures behaves like different sensors [4,5].

Sensor operating temperature is, therefore, a tunable parameter that can be selected for an optimized sensor performance. Computational methodologies have been presented for temperature waveform optimization to increase the selectivity between methanol and ethanol [6], to select the most effective temperature modulation frequency [7], and to reduce power consumption in real time [8]. However, these optimization approaches do not quantify the ability of the sensors in odor quality classification according to the operating temperature of the sensors.

Information theory tools have been proposed to concisely quantify the performance estimation of sensor arrays according to the combined response of the sensors and a control variable [9]. More recently, Vergara et al. [10] presented a method based on the Kullback-Leibler distance to select the best operating temperature of a single sensor to discriminate a set of odorants.

In this paper we propose a methodology based on Mutual Information (MI) maximization to select the operating temperatures of arrays of MOX gas sensors for an efficient odor discrimination task.

\section{Mutual Information applied to gas sensing}

Information theory was developed by Shannon to measure the efficiency of communication systems and provide tools to describe data transmission [11]. The entropy (S) is a quantitative measurement of the disorder of a system and describes the uncertainty in defining the state of a random variable $Y$. The number of possible states $(N)$ of the variable $Y$ and the corresponding probability of occurrence determine the system entropy $S$ (in units of bits): 
$S=-\sum_{i=1}^{N} p\left(y_{i}\right) \log _{2} p\left(y_{i}\right)$

The higher the entropy, the more challenging is the prediction of the state of the variable $\mathrm{Y}$. However, the information provided by the known state of another random variable $X$ may contribute to disambiguate the state of the system.

Mutual Information (MI) is a quantitative measurement of the amount of information of one random variable $(Y)$ contained into another random variable $(X)$.

Thus, MI quantifies the reduction of uncertainty of the variable $Y$ when the state of the variable $X$ is known. $\mathrm{Ml}$ can be calculated by means of the marginal probability distribution functions $p x(i)$ and $p y(j)$ and the joint probability distribution function $p(i, j)[12]$ :

$$
M I=\sum_{i, j} p(i, j) \log _{2} \frac{p(i, j)}{p_{x}(i) p_{y}(j)}
$$

If $X$ and $Y$ are completely independent variables, the state of $X$ does not provide any information on the state of the variable $Y$ and $\mathrm{MI}$ is 0 . On the contrary, if $X$ and $Y$ are coincident, the state of variable $X$ allows perfect attainment of the state of variable $\mathrm{Y}$ and $\mathrm{Ml}$ is equal to the entropy of the system.

In this work we have applied MI to quantify the performance of different sets of sensors in two different tasks: gas concentration prediction and odorant discrimination. Therefore, MI shows the ability of the sensor array to predict the concentration of an already defined volatile or to determine the quality of the odorant.

\section{Sensor models}

We measured the resistance of TGS-2620 and TGS-2600 MOX sensors (Figaro Inc.) and SB15-00 and SB-11-00 MOX sensors (FIS Inc.) using a simple signal conditioning circuit based on a linear voltage divider supplied at $10 \mathrm{~V}$ with a load resistance of $3.01 \mathrm{~K} \Omega$. We applied 94 different voltages on the heaters and exposed the sensors to ethanol, acetone, 2-butanone and acetic acid at 5 different concentration levels in the range of $12 \mathrm{ppm}$ and $160 \mathrm{ppm}$.

Fig. 1 shows the measured voltage when a TGS-2620 sensor is exposed to air, 162ppm of ethanol, $135 \mathrm{ppm}$ of acetone, $113 \mathrm{ppm}$ of 2butanone, and $175 \mathrm{ppm}$ of acetic acid for the 94 different operating temperatures. From Fig. 1, we can conclude that MOX gas sensors show a particular pattern of response for every gas, and, moreover, the sensitivity of MOX sensors depends on the sensor temperature.

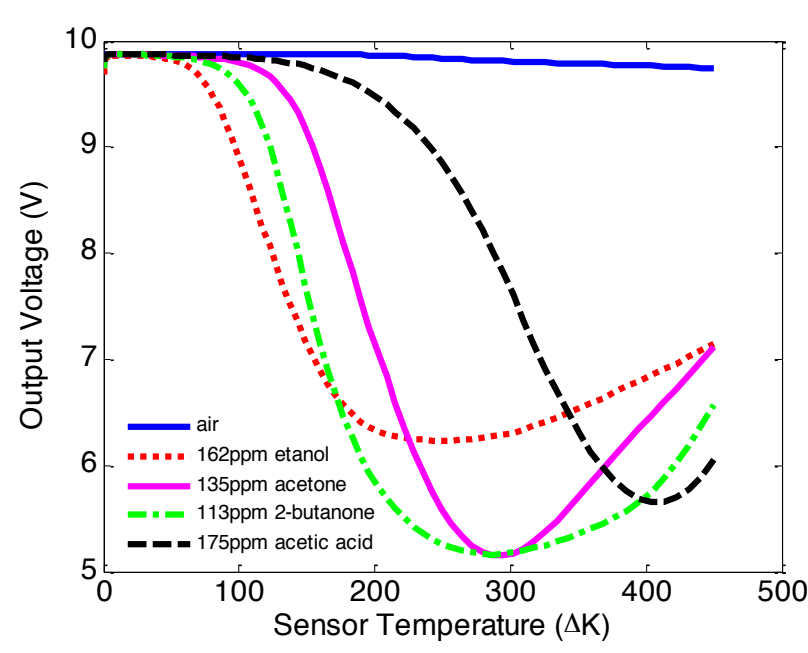

Fig. 1. Measured voltage when exposing a TGS2620 sensor to different gas conditions and operating at different temperature.

The Clifford-Tuma model $[13,14]$ relates the sensor resistance when it is exposed to pure air $R_{\text {air }}$ with the resistance $R_{s}$ when a gas is present:

$$
\frac{R_{\text {air }}(T)}{R_{S}(T)}=1+S(T) c^{\beta(T)}
$$

where $S$ is the sensitivity to the gas, $T$ is the operating temperature of the sensor, $c$ is the gas concentration, and $\beta$ is a gas depending parameter with values around 0.5. The resistance variation is, therefore, a particular function for each sensor but also depends on the measuring gas and the sensor temperature.

Using the experimental data we built complete models for each sensor by fitting eq. (3) and assuming $\mathrm{Sc}^{\beta}>>1$. The result is a complete temperature-dependent model for the four different types of sensors to shape the sensor response to the different gases. Fig. 2 shows the measured resistance of a TGS2620 sensor heated at $\Delta T=402 \mathrm{~K}$ under different gas conditions and the corresponding sensor model.

\section{Results}

\section{Odor concentration prediction}

We tested the ability of a single TGS2620 sensor to predict the gas concentration by means of the MI theory and using the corresponding sensor model. We assumed that the sensor is exposed only to one gas, which is known in advance, and we quantified the ability of the sensor to predict its concentration using the MI.

We built a random vector to represent different gas concentrations in the range of $5-300 \mathrm{ppm}$ 
according to a uniform distribution and we estimated the sensor voltage for each stimulus by means of the sensor model. We added a $50 \mathrm{mV}$ Gaussian white noise to the output voltage and we simulated the voltage acquisition with an ADC with a resolution of 8 bits. Finally, we calculated the MI between the gas concentration (stimuli) vector and the acquired sensor voltage (response). We repeated the routine for ethanol, acetone, 2butanone and acetic acid and for all the set of temperatures.

Fig. 3 shows the MI, which is limited to the resolution of the ADC (i.e. 8 bits) when the sensor is operating at different temperatures.

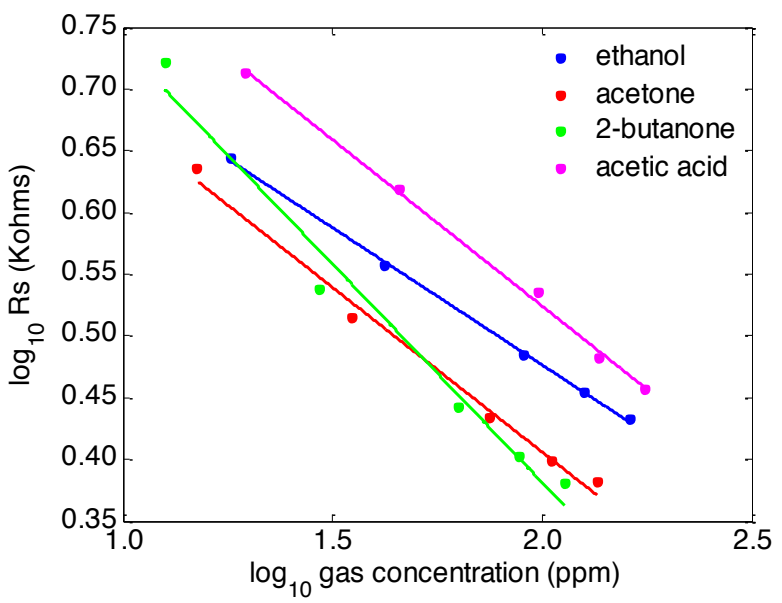

Fig. 2. Measured TGS2620 sensor resistance (dots) under different gas concentration levels of ethanol, acetone, 2-butanone, and acetic acid when the sensor is at $\Delta T=402 \mathrm{~K}$. The corresponding theoretical model is built fitting eq. 3 (solid lines).

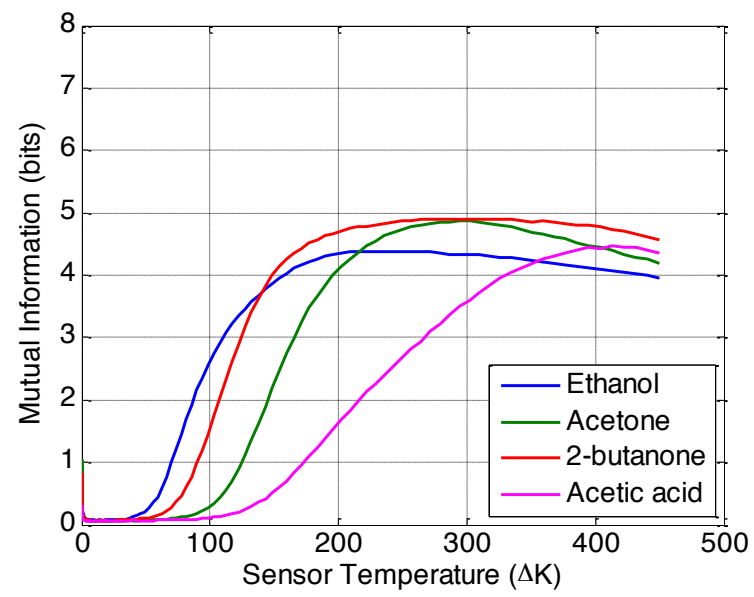

Figure 3: Ability of a TGS2620 sensor to predict the gas concentration in the range of 5-300ppm when operating at different temperatures.

The sensor performance at low temperatures is inefficient and, therefore, the MI yields to zero. On the other extreme, there is an operating temperature $\Delta T_{0}$ that shows optimal gas concentration prediction. $\Delta T_{0}$ depends on the gas to measure and we obtained $\Delta \mathrm{T}_{0}=229 \mathrm{~K}$, $\Delta \mathrm{T}_{0}=294 \mathrm{~K}, \Delta \mathrm{T}_{0}=318 \mathrm{~K}$, and $\Delta \mathrm{T}_{0}=413 \mathrm{~K}$ for ethanol, acetone, 2-butanone, and acetic acid respectively. Moreover, the dependency of the sensor performance against the temperature is sharper for acetic acid and acetone than for ethanol and 2-butanone.

We can conclude, therefore, that the optimal operating temperature depends on both the sensor temperature, which is controlled by applying a voltage on the heater, and on the target gas.

\section{Odor discrimination task}

In a four odor discrimination task, if we assume an equal probability of occurrence for all the chemicals, the system entropy is 2 bits (four different states equally likely).

We explored the ability of a pair of sensors (SB15-00 and TGS 2600) in a 4-odorant discrimination task by measuring the MI. In this task the purpose of the system is to identify the quality of the stimulus regardless its concentration.

We used the developed sensor models, we simulated the system acquisition with an 8 bits resolution $A D C$ and limited the concentration in the range $0.1-1000 \mathrm{ppm}$ for all the gases. Finally, we calculated the Ml between the quality of the gas at the input and the combined response of the sensors.

In order to find the optimal pair of operating points we calculated the MI for the two sensors at different temperatures. Fig. 4 shows the 2sensor array performance in a discrimination task of 4 gases for the $94^{2}$ combinations of different operating temperatures. The optimal pair of operating temperatures corresponds to $\Delta \mathrm{T}_{\mathrm{SB}-15-00}=112 \mathrm{~K}$ and $\Delta \mathrm{T}_{\mathrm{TGS} 2600}=394 \mathrm{~K}$, which yields a $\mathrm{Ml}=0.83$.

We evaluated to what extent the discrimination task is improved by introducing two new sensors into the array. We simulated the ability of the 4-sensor array composed of TGS2620, TGS2600, SB-15-00 and SB-11-00 sensors in the discrimination task of four gases.

We calculated the $\mathrm{MI}$ for a $94^{4}$ combinations of different sensor temperatures. In this conditions, the optimal sensor temperatures are $\Delta \mathrm{T}_{\mathrm{SB}-15-00}=334 \mathrm{~K}, \Delta \mathrm{T}_{\mathrm{TGS} 2600}=450 \mathrm{~K}, \Delta \mathrm{T}_{\mathrm{TGS} 2620}=$ $202 \mathrm{~K}$, and $\Delta \mathrm{T}_{\mathrm{SB}-11-00}=310 \mathrm{~K}$ that correspond to $\mathrm{MI}=1.23$. Therefore, the ability of the sensor array increases from $\mathrm{Ml}=0.83$ to $\mathrm{Ml}=1.23$ when introducing SB-11-00 and TGS2620 sensors to the array. It is important to note that the optimal temperatures of the TGS2600 and SB-15-00 
sensors do not correspond to the same temperatures when working together with other two sensors.

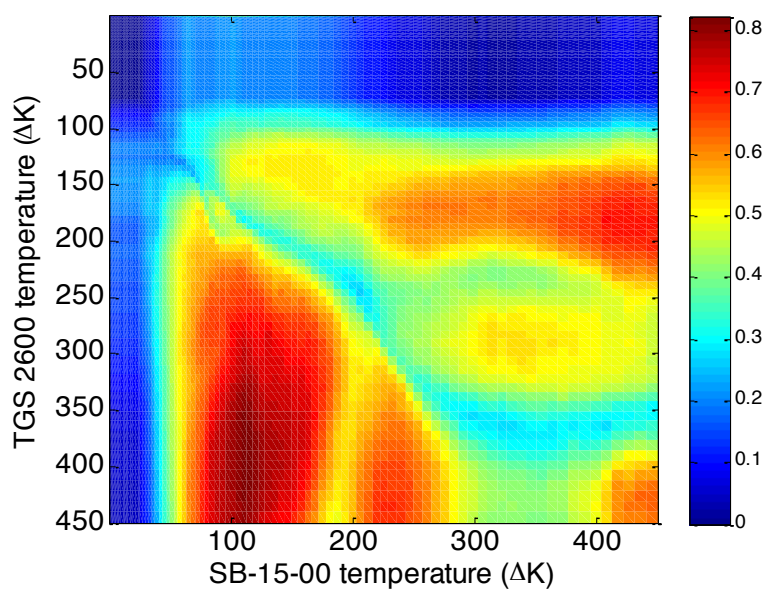

Fig. 4: Mutual Information (in bits) of SB-15-00 and TGS 2600 sensors to discriminate among ethanol, acetic acid, 2-butanone, and acetone.

\section{Conclusions}

We presented a method based on the maximization of the Mutual Information to optimize the performance of sensor arrays in the discrimination of different odorants. We applied the methodology to find the optimal sensor operating temperatures of a four-sensor array and quantified the system ability to discriminate ethanol, acetone, acetic acid and 2-butanone.

\section{Acknowledgements}

The research leading to these results has received funding from the European Community's Seventh Framework Programme (FP7/2007-2013) under grant agreement no.216916.

The authors are from a consolidated Grup de Recerca de la Generalitat de Catalunya and has support from the Departament d'Universitats, Recerca I Societat de la Informació la Generalitat de Catalunya (expedient 2009 SGR 0753)

\section{References}

[1] Barsan N, Koziej D, Weimar U (2007) Metal oxide-based gas sensor research: How to? Sensors and Actuators B-Chemical 121: 18-35.

[2] Korotcenkov G (2007) Metal oxides for solid-state gas sensors: What determines our choice? Materials Science and Engineering B-Solid State Materials for Advanced Technology 139: 1-23.

[3] Elmi I, Zampolli S, Cardinali GC (2008) Optimization of a wafer-level process for the fabrication of highly reproducible thin-film MOX sensors. Sensors and Actuators B-Chemical 131: 548-555.

[4] Lee AP, Reedy BJ (1999) Temperature modulation in semiconductor gas sensing. Sensors and Actuators B-Chemical 60: 35-42.

[5] Fort A, Gregorkiewitz M, Machetti N, Rocchi S, Serrano B, et al. (2002) Selectivity enhancement of $\mathrm{SnO} 2$ sensors by means of operating temperature modulation. Thin Solid Films 418: 28.

[6] Kunt TA, McAvoy TJ, Cavicchi RE, Semancik S (1998) Optimization of temperature programmed sensing for gas identification using micro-hotplate sensors. Sensors and Actuators B-Chemical 53: 24-43.

[7] Vergara A, Llobet E, Brezmes J, Ivanov P, Cane C, et al. (2007) Quantitative gas mixture analysis using temperature-modulated micro-hotplate gas sensors: Selection and validation of the optimal modulating frequencies. Sensors and Actuators B-Chemical 123: 1002-1016.

[8] Gosangi R, Gutierrez-Osuna R (2010) Active Temperature Programming for Metal-Oxide Chemoresistors. leee Sensors Journal 10: 10751082.

[9] Pearce T, Sanchez-Montañes A (2002) Chemical Sensor Array Optimization: Geometric and Information Theoretic Approaches Handbook of Artificial Olfaction Machines: Wiley$\mathrm{VCH}$, Weinheim.

[10] Vergara A, Muezzinoglu MK, Rulkov N, Huerta R (2010) Information-theoretic optimization of chemical sensors. Sensors and Actuators BChemical 148: 298-306.

[11] Shannon CE (1948) A Mathematical Theory Of Communication. Bell System Technical Journal 27: 379-423.

[12] Moddemeijer R (1999) A statistic to estimate the variance of the histogram-based mutual information estimator based on dependent pairs of observations. Signal Processing 75: 51-63.

[13] Clifford PK, Tuma DT (1983) Characteristics of semiconductor gas sensors .1. Steady-state gas response. Sensors and Actuators 3: 233-254.

[14] Clifford PK, Tuma DT (1983) Characteristics of semiconductor gas sensors .2. Transientresponse to temperature-change. Sensors and Actuators 3: 255-281 